\title{
Mass renormalization in string theory: special states
}

\author{
Roji Pius, ${ }^{a}$ Arnab Rudra ${ }^{b}$ and Ashoke Sen ${ }^{a}$ \\ ${ }^{a}$ Harish-Chandra Research Institute, \\ Chhatnag Road, Jhusi, Allahabad 211019, India \\ ${ }^{b}$ Department of Applied Mathematics and Theoretical Physics,, \\ Wilberforce Road, Cambridge CB3 OWA, U.K. \\ E-mail: rojipius@mri.ernet.in, A.Rudra@damtp.cam.ac.uk, \\ sen@mri.ernet.in
}

ABSTRACT: String theory gives a well defined procedure for computing the S-matrix of BPS or a class of massless states, but similar calculation for general massive states is plagued with difficulties due to mass renormalization effect. In this paper we describe a procedure for computing the renormalized masses and S-matrix elements in bosonic string theory for a special class of massive states which do not mix with unphysical states under renormalization. Even though this requires working with off-shell amplitudes which are ambiguous, we show that the renormalized masses and S-matrix elements are free from these ambiguities. We also argue that the masses and S-matrix elements for general external states can be found by examining the locations of the poles and the residues of the S-matrix of special states. Finally we discuss generalizations to heterotic and superstring theories.

Keywords: Superstrings and Heterotic Strings, Bosonic Strings

ARXIV EPRINT: 1311.1257 


\section{Contents}

1 Introduction 1

2 The question $\quad 2$

3 Mass renormalization $\quad 6$

3.1 Analysis of poles of off-shell two point function 8

$\begin{array}{ll}3.2 & \text { Explicit construction of } \widetilde{F} \text { and } \delta \widetilde{H} \\ \end{array}$

4 S-matrix elements $\quad 15$

$\begin{array}{llr}5 & \text { Discussion and generalizations } & 18\end{array}$

\section{Introduction}

String theory does not have a non-perturbative definition at present, but it gives a well defined procedure for computing S-matrix elements involving BPS or a class of massless external states - whose masses are protected from renormalization - to any order in perturbation theory. Furthermore this perturbation expansion is free from ultraviolet divergences (see e.g. [1-17] for recent discussion). However the usual procedure for computing S-matrix elements breaks down for general massive states. This is due to the fact that for general massive states loop corrections generate (ultraviolet finite) mass renormalizations, and hence in order to compute the physical S-matrix elements we have to shift the external momenta to their renormalized on-shell values. On the other hand string perturbation theory, which is based on world-sheet conformal invariance, requires the vertex operators representing external states to be dimension zero primary fields. This is equivalent to requiring that the external momenta satisfy the tree level on-shell condition.

For many states this is not a problem since they appear as single particle intermediate states in the S-matrix of massless and/or BPS external states and hence their renormalized masses and S-matrix elements can be found by examining the locations and residues of the poles of the S-matrix of massless and/or BPS states. For this reason direct computation of the S-matrix of massive string states has not received much attention. However this does not always work, e.g. if the massive state under consideration carries a conserved charged that is not carried by any of the massless or BPS states, then the former cannot appear as a single particle intermediate state in the S-matrix of the latter. For this reason it seems important to find a more direct approach to computing the mass renormalization and S-matrix elements of massive string states.

In this paper we undertake this task for a special class of states in bosonic string theory. There are two related but independent problems which arise in the computation 
of mass renormalization in string theory. First we have to define the analog of the offshell Green's function in string theory. This requires giving up the conformal invariance of vertex operators and hence is ambiguous. Second, under renormalization the BRST invariant physical states begin mixing with the unphysical states of string theory and hence the definition of the physical state needs to be modified carefully. By choosing a special class of states we avoid the second problem - these special class of states do not mix with unphysical states due to some global symmetries. However we still need to deal with the first problem, 1.e. the ambiguity in the definition of the off-shell Greens function. We show that although the off-shell Greens functions are ambiguous, the renormalized mass and S-matrix elements computed from them are free from these ambiguities.

The paper is organised as follows. In section 2 we make precise the problem associated with mass renormalization in string theory, and also introduce the special class of states for which we address the problem in this paper. In section 3 we describe how to compute the renormalized mass of these special states and also show that this renormalized mass is free from any ambiguity. In section 4 we show how to compute the S-matrix elements of these special states, and demonstrate that they are also free from ambiguities. We end in section 5 with a discussion of our results, extensions to heterotic and superstring theories and future generalizations.

Various other approaches to studying mass renormalization in string theory can be found in [18-31]

\section{The question}

Consider a string theory amplitude with $n$-external states representing particles carrying momenta $k_{1}, \cdots k_{n}$ and other discrete quantum numbers $a_{1}, \cdots a_{n}$ with tree level masses $m_{a_{1}}, \cdots m_{a_{n}}$. Then the momenta $k_{i}$ satisfy the tree level on-shell condition $k_{i}^{2}=-m_{a_{i}}^{2}$, - this is needed to ensure the BRST invariance of the vertex operators in the world sheet theory. The world-sheet computation, involving correlation functions of these vertex operators integrated over the moduli spaces of (punctured) Riemann surfaces, yields the result for what in a quantum field theory can be called 'truncated Green's function on classical mass shell': ${ }^{1}$

$$
\begin{aligned}
& R_{a_{1} \cdots a_{n}}^{(n)}\left(k_{1}, \cdots k_{n}\right) \equiv \lim _{k_{i}^{2} \rightarrow-m_{a_{i}}^{2}} F_{a_{1} \cdots a_{n}}^{(n)}\left(k_{1}, \cdots k_{n}\right), \\
& F_{a_{1} \cdots a_{n}}^{(n)}\left(k_{1}, \cdots k_{n}\right) \equiv G_{a_{1} \cdots a_{n}}^{(n)}\left(k_{1}, \cdots k_{n}\right) \prod_{i=1}^{n}\left(k_{i}^{2}+m_{a_{i}}^{2}\right),
\end{aligned}
$$

where $G_{a_{1} \cdots a_{n}}^{(n)}\left(k_{1}, \cdots k_{n}\right)$ correspond to the momentum space Green's function in the quantum field theory. This is similar to but not the same as the combination that appears in the expression for the S-matrix in a quantum field theory

$$
S_{a_{1} \cdots a_{n}}^{(n)}\left(k_{1}, \cdots k_{n}\right)=\lim _{k_{i}^{2} \rightarrow-m_{a_{i}, p}^{2}} G_{a_{1} \cdots a_{n}}^{(n)}\left(k_{1}, \cdots k_{n}\right) \prod_{i=1}^{n}\left\{Z^{-1 / 2}\left(k_{i}, a_{i}\right)\left(k_{i}^{2}+m_{a_{i}, p}^{2}\right)\right\},
$$

\footnotetext{
${ }^{1}$ We have absored all factors of $\mathbf{i} \equiv \sqrt{-1}$ and minus signs into the definition of $G^{(n)}$ and $R^{(n)}$.
} 
where $m_{a_{i}, p}$ is the physical mass of the $i$-th particle, defined as the location of the pole as a function of $-k^{2}$ in the untruncated two point Green's function $G^{(2)}$ and $Z\left(k_{i}, a_{i}\right)$ 's are the residues at these poles.

For simplicity we have ignored the mixing between different states under wave-function renormalization in writing down (2.2), but we shall discuss the general case now. If we consider the set of all fields whose tree level masses are all equal to $m$ then the two point Green's function $G_{a b}^{(2)}\left(k, k^{\prime}\right)$ for all these fields is described by the matrix

$$
G_{a b}^{(2)}\left(k, k^{\prime}\right)=(2 \pi)^{D+1} \delta^{(D+1)}\left(k+k^{\prime}\right) Z^{1 / 2}(k)_{a c}\left(k^{2}+M_{p}^{2}\right)_{c d}^{-1}\left(Z^{1 / 2}(-k)\right)_{d b}^{T},
$$

where $M_{p}^{2}$ is the mass ${ }^{2}$ matrix and $Z^{1 / 2}(k)$ is the wave-function renormalization matrix, the latter being free from poles near $k^{2}+m^{2} \simeq 0$. The sum over $c, d$ are restricted to states which have the same tree level mass $m$ as the states labelled by the indices $a, b . D+1$ is the total number of non-compact space-time dimensions. We can diagonalize $M_{p}^{2}$ and absorb the diagonalizing matrices into the wave-function renormalization factor $Z^{1 / 2}(k)$ to express $M_{p}^{2}$ as a diagonal matrix. These eigenvalues, which we shall denote by $m_{a, p}^{2}$, are the squares of the physical masses. Taking into account the non-diagonal nature of the wave-function renormalization factor $Z,(2.2)$ is modified to

$$
S_{a_{1} \cdots a_{n}}^{(n)}\left(k_{1}, \cdots k_{n}\right)=\lim _{k_{i}^{2} \rightarrow-m_{a_{i}, p}^{2}} G_{b_{1} \cdots b_{n}}^{(n)}\left(k_{1}, \cdots k_{n}\right) \prod_{i=1}^{n}\left\{Z_{i}^{-1 / 2}\left(k_{i}\right)_{a_{i}, b_{i}}\left(k_{i}^{2}+m_{a_{i}, p}^{2}\right)\right\},
$$

where $Z_{i}^{-1 / 2}$ is the inverse of the matrix $Z^{1 / 2}$ introduced in (2.3) for the $i$-th external state. In this expression we can interpret the sum over $b_{i}$ 's as sum over all fields in the theory if we define $Z^{1 / 2}(k)_{a b}$ and $Z^{-1 / 2}(k)_{a b}$ to be zero when $a, b$ label fields with different classical mass.

At tree level $Z=1, M_{p}^{2}=m^{2} I$ and hence the $R^{(n)}$ defined in (2.1) and $S^{(n)}$ defined in (2.4) agree. In general however $R^{(n)}$ and $S^{(n)}$ are different. While $S^{(n)}$ defined in (2.4) is the physically relevant quantity, string theory directly computes $R^{(n)}$ defined in (2.1). Thus the question arises: how can we use string theory to compute on-shell S-matrix elements beyond tree level? At a more basic level: how can we use string theory to calculate the physical mass $m_{a_{i}, p}$ of the $i$-th particle?

When the external strings represent massless gauge particles, the situation improves dramatically. In this case gauge symmetry prevents mass renormalization and hence we have $m_{a_{i}, p}^{2}=m_{a_{i}}^{2}=0$. As a result $R^{(n)}$ and $S^{(n)}$ differ only by the wave-function renormalization factor $Z$. This can be fixed by using analyticity property of the S-matrix, e.g. the S-matrix should factorize into the product of lower point S-matrices when the external momenta are such that some internal line could become on-shell. Thus string world-sheet computation can be used to compute the S-matrix of massless external states.

Now typically in string theories many massive string states appear as one particle intermediate states in the scattering of massless states and as a result the S-matrix of the massless states can have poles when the square of appropriate combination of external momenta approaches the squared mass of a massive state. The location of this pole gives information about the mass of the massive state while the residue at this pole contains 
information about the S-matrix involving massive external states. However this procedure does not always work. Some string theories contain massive states which do not appear as one particle intermediate states in the scattering of massless particles. We shall now describe some examples of such situations.

1. Consider bosonic string theory compactifed on a circle $S^{1}$. In this case a state carrying a winding number (and/or momentum) along $S^{1}$ cannot be produced as single particle intermediate state in the scattering of massless states which do not carry any momentum and winding charge. ${ }^{2}$

2. Another notable example is $\mathrm{SO}(32)$ heterotic string theory which contains massive states belonging to the spinor representation of $\mathrm{SO}(32)$. They cannot appear as single particle intermediate states in the scattering of massless external states which are all in the adjoint or singlet representation of $\mathrm{SO}(32)$. Thus the S-matrix element involving these particles cannot be computed by examining any massless S-matrix element near its poles.

In order to deal with these cases we shall try to develop a different strategy — compute the mass renormalization directly. We shall focus on a special class of states - which we shall call special states - for which the analysis simplifies. We shall conclude this section by describing these special states and their relevance to the problems mentioned above.

Let us suppose that we are dealing with a string theory with $D+1$ non-compact dimensions, with $\mathrm{SO}(D, 1)$ Lorentz invariance. Then while discussing the mass renormalization of a massive state we can go to the rest frame of the particle so that the spatial component $\vec{k}$ of the momentum vanishes. In this frame we consider physical states described by vertex operators of the form

$$
c \bar{c} e^{ \pm \mathbf{i} k_{0} X^{0}} V
$$

where $c, \bar{c}$ are ghost fields and $V$ is a dimension $(h, h)$ primary made of the compact coordinates and the oscillators of the non-compact spatial coordinates. The on-shell condition on $k_{0}$ is

$$
k^{0}=m, \quad m^{2}=4(h-1),
$$

in the $\alpha^{\prime}=1$ units. The operators $V$ will form a finite dimensional representation of the $\mathrm{SO}(D)$ little group. If the world-sheet theory has additional global symmetry group $G$ associated with the compact directions then the operators $V$ will also belong to finite dimensional representation of this symmetry group.

Now consider all operators of the form $e^{ \pm \mathbf{i} k_{0} X^{0}} \mathcal{O}$ where $\mathcal{O}$ 's are dimension $(h-1, h-1)$ operators made of the ghost fields, compact coordinates and oscillators of $X^{0}$ and the non-compact spatial coordinates. They can be organised into irreducible representations of $\mathrm{SO}(D) \times G$. Among them the operators which are not of the form (2.5) will be called

\footnotetext{
${ }^{2}$ Such states could still appear in pairs in the intermediate channel, producing a cut in the S-matrix of massless states, and by examining where the cut begins, we can find the mass of the intermediate state. But it is much harder to identify cuts than poles in the S-matrix, and we shall not explore this option.
} 
unphysical vertex operators at mass level $m .^{3}$ We shall define special vertex operators to be a set of vertex operators of the form given in (2.5) belonging to those irreducible representations of the symmetry group $\mathrm{SO}(D) \times G$ such that there are no unphysical vertex operator at mass level $m$ transforming in these representations. Put another way, if the unphysical vertex operators at mass level $m$ transform in certain irreducible representations $R_{1}, R_{2}, \cdots$ then the special vertex operators are those physical states which transform in representations other than $R_{1}, R_{2}, \cdots$. In this case the two point function of any special vertex operator and an unphysical operator on any Riemann surface will vanish.

We shall now give some examples of special vertex operators.

1. Consider bosonic string theory in $25+1$ dimensions. We consider vertex operators of the form (2.5) with $V$ given by

$$
S\left[\partial X^{i_{1}} \cdots \partial X^{i_{n}} \bar{\partial} X^{j_{1}} \cdots \bar{\partial} X^{j_{n}}\right] .
$$

where $X^{i}$ for $1 \leq i \leq 25$ are the spatial coordinates and $S$ denotes the operation of taking the symmetric traceless part of the product. This belongs to a rank $2 n$ symmetric traceless representation of $\mathrm{SO}(25)$ - also known as the leading Regge trajectory. In order to get an unphyical state at the same mass level we have to replace some of the $\partial X^{i}$ or $\bar{\partial} X^{j}$ by ghost or $X^{0}$ excitations and/or replace the product of some of the $\partial X^{i}$ 's and/or $\bar{\partial} X^{i}$ 's by higher derivatives of $X^{i}$ 's. This clearly reduces the rank of the tensor and hence the unphysical states cannot belong to the rank $2 n$ symmetric tensor representation of $\mathrm{SO}(25)$. Thus vertex operators of the form (2.7) are special.

2. Consider bosonic string theory compactified on a circle. Let $Y$ be the coordinate along the compact direction, and $Y_{L}$ and $Y_{R}$ be its left and right-moving components on the world-sheet. We now consider the vertex operator of the form (2.5) with

$$
V=e^{ \pm \mathbf{i}(n / R-w R) Y_{L} / 2} e^{ \pm \mathbf{i}(n / R+w R) Y_{R} / 2} S\left[\bar{\partial} X^{i_{1}} \cdots \bar{\partial} X^{i_{p}} \partial X^{j_{1}} \cdots \partial X^{j_{q}}\right], \quad p-q=n w,
$$

where $X^{i}$ for $i=1, \cdots 24$ denote the non-compact directions and $S$ stands for the projection into rank $p+q$ symmetric traceless representation of $\mathrm{SO}(24)$. Following the same argument as before it follows that there are no unphysical vertex operators at this mass level carrying $n$ units of momentum, $w$ units of winding and belonging to the rank $p+q$ symmetric traceless representation of $\mathrm{SO}(24)$. Thus these are also special states according to the definitions given above.

3. Finally we note that the stable non-BPS states of $\mathrm{SO}(32)$ heterotic string theory which correspond to the lowest mass states in the spinor representation of $\mathrm{SO}(32)$ - are also special states. Besides the ghost fields and the $e^{ \pm \mathbf{i} k_{0} X^{0}}$ factor, the leftmoving part of the vertex operator is given by the $\mathrm{SO}(32)$ spin field of dimension

\footnotetext{
${ }^{3}$ Technically the unphysical operators described here can the divided into two kinds, BRST trivial ones and states which are not invariant under BRST transformation. The former are called pure gauge and the latter are called unphysical. We shall not need to make this distinction, and call all such states unphysical.
} 
2, and has no further oscillator excitations. Level matching requires that the rightmoving part of the Neveu-Schwarz (NS) sector vertex operator corresponds to level $3 / 2$ excitations above the NS-sector ground state. We can take this to be $\psi^{i} \psi^{j} \psi^{k}$ where $\psi^{i}$ for $1 \leq i \leq 9$ are the world-sheet superpartners of the 9 non-compact bosonic coordinates. This belongs to the totally anti-symmetric rank 3 tensor representation $\mathbf{8 4}$ of $\mathrm{SO}(9)$. It is easy to see that any other unphysical state at this mass level, obtained by replacing some of the $\psi^{i}$ 's by ghost or $\psi^{0}$ oscillators or derivatives of $\psi^{i}$ or bosonic coordinates cannot belong to the $\mathbf{8 4}$ representation of $\mathrm{SO}(9)$. Thus these states are special states.

The reader might have noticed that there is a close relationship between special states which are prevented from mixing with the unphysical states due to global symmetry on the world-sheet and the states which cannot appear as poles in the scattering of massless states due to conserved charges. Indeed the lowest mass states in each of the examples of the latter kind given earlier also correspond to special states. On general grounds one expects that in every charge sector we can construct a set of special states by saturating the required oscillator levels by (anti-)symmetric products of bosonic (fermionic) fields associated with the non-compact coordinates. For this reason we shall focus on computation of physical mass and S-matrix elements involving these special states and massless states, since the renormalized mass and S-matrix elements of all other states can be obtained from the locations of the poles of the S-matrix involving the special states and massless states.

\section{Mass renormalization}

If we work in the rest frame, then the off-shell continuation of a special vertex operator would correspond to deforming $k^{0}$ away from $m$. This keeps the vertex operator primary but it no longer has dimension 0 . Thus in order to define the correlation functions of such vertex operators on a Riemann surface we need to make a choice of local coordinate around every point on the Riemann surface. If $z$ denotes some reference cordinate system on the Riemann surface then the local coordinate $w$ around some point $z=z_{0}$ is described by some function $f\left(w ; z_{0}\right)$ that maps the $w$ plane to the $z$ plane around $z=z_{0}$. We take $f\left(0 ; z_{0}\right)=z_{0}$ and $f\left(w ; z_{0}\right)$ to be analytic around $w=0$. Thus $f$ depends on both, the choice of the reference coordinate system $z$ and the choice of the local coordinate system $w$. The vertex operator at $z_{0}$ is inserted using the local coordinate $w$, which corresponds to inserting its conformal transformation by the function $f\left(w ; z_{0}\right)$ in the $z$ coordinate system [32]. Thus if the off-shell vertex operator is a primary operator of dimension $(\delta, \delta)$ then we multiply it by $\left(f^{\prime}\left(0 ; z_{0}\right)\right)^{\delta}\left(\overline{f^{\prime}\left(0 ; z_{0}\right)}\right)^{\delta}$ while inserting it into the correlation function in the $z$ coordinate system, $f^{\prime}$ being the derivative of the function $f\left(w ; z_{0}\right)$ with respcet to $w$. For more general vertex operator representing general off-shell string state the same procedure would work although the conformal transform of the vertex operator will be more complicated.. ${ }^{4}$ This definition makes the correlation function invariant under a change of reference coordinate system $z$, but dependent on the choice of local coordinates $w$, 1.e. the function $f\left(w ; z_{0}\right)$.

\footnotetext{
${ }^{4}$ For related approaches to defining off-shell amplitudes in string theory, see [33-36]
} 
As a result, if we define off-shell strng amplitudes by integrating such correlation functions over the moduli space, the result will depend on the choice of local coordinates.

In a sense the situation in string theory is not very different from that in a gauge theory. In gauge theory for computing the mass renormalizaton of a massive charged particle we have to first compute the off-shell propagator carrying momentum $k=\left(k^{0}, \overrightarrow{0}\right)$ and then look for its poles in the $k^{0}$ plane. The off-shell propagator is not gauge invariant; however the location of its pole in the $k^{0}$ plane is gauge invariant and leads to a gauge invariant definition of the renormalized mass. Thus a possible strategy in string theory will be to consider off-shell propagator that depends on the choice of local coordinates, look for its poles in the $k^{0}$ plane and prove that the location of the pole is independent of the choice of local coordinates even though the propagator itself is not gauge invariant. If we had an underlying string field theory then this analysis will be parallel to that in an ordinary gauge theory. This can be done in principle for bosonic string theory where a complete closed string field theory is known [37]. ${ }^{5}$ At present there is no known string field theory for closed heterotic and superstring theories except a closed heterotic string field theory at tree level [38]. Nevertheless we can try to extract the relevant features of the off-shell string theory amplitudes from a bosonic string field theory and then develop a general proof of indpendence of the renormalized mass of the choice of local coordinates that does not require the existence of an underlying string field theory. The essential features seem to be the following:

1. Bosonic string field theory gives a triangulation of the punctured Riemann surface equipped with local coordinate system at each puncture. Using this local coordinate system we can define off-shell amplitudes.

2. Near boundaries of the moduli space where a Riemann surface of genus $n$ degenerates into two Riemann surfaces of genus $n_{1}$ and $n_{2}=n-n_{1}$ connected by a long handle, the choice of the local coordinates of the original Riemann surface matches with the choice of the local coordinates of the lower genus surfaces. The precise meaning on 'near boundaries of the moduli space' will be made clear later (see item 6 in the discussion in section 3.2 (above eq. (3.21)).

For an off-shell amplitude induced from string field theory the above requirements are automatically satisfied, but even in the absence of string field theory we could try to choose local coordinates at the punctures consistent with the above criteria. Indeed even before the construction of fully covariant closed string field theory, such choices of local coordinates were explored (see e.g. [39]). Given such a choice of local coordinates, we can define off-shell two point functions in string theory and define the mass to be the location of the pole in the $k^{0}$ plane. The important point is to show that this definition is independent of the choice of local coordinates.

From now on we shall restrict our analysis to bosonic string theory. We discuss possible generalizations to superstring and heterotic string theories in $\S 5$.

\footnotetext{
${ }^{5}$ Due to the presence of tachyon, the mass renormalization in this case is infrared dvergent.
} 


\subsection{Analysis of poles of off-shell two point function}

Let us denote the set of all the special vertex operators by $c \bar{c} V_{i} e^{\mathrm{i} k_{0} X^{0}}$ and the corresponding states as

$$
c_{1} \bar{c}_{1}\left|V_{i}\right\rangle \otimes\left|k^{0}, \vec{k}=0\right\rangle .
$$

In the zero mode sector of non-compact bosons labelled by $\left(k^{0}, \vec{k}\right)$, the states satisfy the usual $\delta$-function normalization. The operaors $V_{i}$ will be chosen so that in the rest of the matter-ghost CFT, they satisfy the orthonormality relation

$$
\left\langle V_{i}\left|c_{-1} \bar{c}_{-1} c_{0} \bar{c}_{0} c_{1} \bar{c}_{1}\right| V_{j}\right\rangle=\delta_{i j}
$$

Let $F(k)$ be the off-shell two point function of special states obtained by summing over all genera. If there are $n_{p}$ special states at mass level $m$ then $F(k)$ is an $n_{p} \times n_{p}$ matrix satifying

$$
F(k)=F(-k)^{T},
$$

where $F^{T}$ denotes transpose of $F$. Then the off-shell propagator of special states is given by ${ }^{6}$

$$
\frac{1}{k^{2}+m^{2}}+\left(\frac{1}{k^{2}+m^{2}}\right)^{2} F(k)
$$

where $m$ is the tree level mass. The first term represents the tree level propagator whereas the first factor of the second term is the effect of the two external propagators. $F(k)$ admits a genus expansion of the form $\sum_{n} F_{n} g^{2 n}$ in string coupling $g$, with higher genus contributions having higher order poles at $k^{2}+m^{2}=0$ from regions of the moduli space where the Riemann surface degenerates into two or more Riemann surfaces of lower genera connected by long handles, with the two external vertices lying on the two lower genus Riemann surfaces at the two ends. We expect that after resummation, (3.4) may be written as $Z^{1 / 2}(k)\left(k^{2}+M_{p}^{2}\right)^{-1}\left(Z^{1 / 2}(-k)\right)^{T}$ for some physical mass ${ }^{2}$ matrix $M_{p}^{2}$ and wavefunction renormalization matrix $Z^{1 / 2}(k)$ which has no pole near $k^{2}=-m^{2}$. This will be seen explicitly in (3.11)-(3.15) below. We can take $M_{p}^{2}$ to be diagonal by absorbing the diagonalizing matrix into the definition of $Z^{1 / 2}(k)$. If $m_{a, p}^{2}$ for $a=1,2 \cdots n_{p}$ are the eigenvalues of the mass ${ }^{2}$ matrix $M_{p}^{2}$ then the physical poles of the propagator are at $k^{2}=-m_{a, p}^{2}$.

Now consider the effect of changing the local coordinate system by an infinitesimal amount. Let the change in $F$ to first order be $\delta F$. Then in order that the location of the poles of the propagator in the $k^{2}$ plane does not shift, the net change in (3.4) must be of the form of an overall multiplicative factor that renormalizes $Z^{1 / 2}(k)$. Thus we require

$$
\begin{aligned}
\frac{1}{k^{2}+m^{2}}+ & \left(\frac{1}{k^{2}+m^{2}}\right)^{2}(F(k)+\delta F(k)) \\
& =(1+\delta Y(k))\left\{\frac{1}{k^{2}+m^{2}}+\left(\frac{1}{k^{2}+m^{2}}\right)^{2} F(k)\right\}(1+\delta Y(-k))^{T},
\end{aligned}
$$

\footnotetext{
${ }^{6}$ We have removed an overall factor of $-\mathbf{i}$ and also absorbed a factor of $-\mathbf{i}$ into the definition of $F(k)$.
} 
for some matrix $\delta Y(k)$ whose genus expansion is free from any poles at $k^{2}+m^{2}=0$. Equivalently we can write

$$
\delta F(k)=\left(k^{2}+m^{2}\right) \delta Y(k)+\left(k^{2}+m^{2}\right)(\delta Y(-k))^{T}+\delta Y(k) F(k)+F(k) \delta Y(-k)^{T} .
$$

At each genus the two point function $\delta F$ receives two contributions - from the change of local coordinates at the vertex carrying momentum $k$ and the change in local coordinates at the vertex carrying momentum $-k$. Both these contributions have an explicit factor of $k^{2}+m^{2}$ due to the fact that when $k^{2}+m^{2}=0$ the vertex is on-shell and hence there is no dependence on local coordinates. In concrete terms, since the off-shell vertex operator of a special state is a primary of dimension $\left(\left(k^{2}+m^{2}\right) / 4,\left(k^{2}+m^{2}\right) / 4\right)$, if we insert such an operator at the origin $w=0$ of the local cordinate system, and then change the local coordinate from $w$ to $w+\epsilon(w)$ then we pick up a net multiplicative factors of $\left(1+\epsilon^{\prime}(0)\right)^{\left(k^{2}+m^{2}\right) / 2} \simeq\left(1+\left(k^{2}+m^{2}\right) \epsilon^{\prime}(0) / 2\right)$. Thus we introduce the function $\delta H$ via the relations

$$
\delta F(k)=\left(k^{2}+m^{2}\right) \delta H(k)+\left(k^{2}+m^{2}\right)(\delta H(-k))^{T},
$$

where the first term is the effect of the change of local coordinates at the vertex carrying momentum $k$ and the second term is the effect of change of local coordinates at the vertex carrying momentum $-k$. The rules for computing $\delta H$ are the same as that of $F$ except that at one of the punctures the vertex $c \bar{c} V_{i}$ is replaced by $\epsilon^{\prime}(0) c \bar{c} V_{i} / 2$. We shall call the puncture where the effect of change of local coordiantes is inserted the 'special puncture'. Eq. (3.6) can now be satisfied by choosing $\delta Y(k)$ such that

$$
\delta H(k)=\delta Y(k)+\left(k^{2}+m^{2}\right)^{-1} \delta Y(k) F(k) .
$$

Our goal will be to show the existence of $\delta Y(k)$ satisfying (3.8) such that the genus expansion of $\delta Y(k)$ does not have any pole at $k^{2}+m^{2}=0$.

We now claim that there exist quantities $\widetilde{F}$ and $\delta \widetilde{H}$ with the properties that the genus expansion of neither of them has any poles near $k^{2}+m^{2}=0$, both have genus expansion starting at one loop and $F$ and $\delta H$ can be expressed in terms of $\widetilde{F}$ and $\delta \widetilde{H}$ as

$$
\begin{aligned}
F & =\widetilde{F}\left(1-\left(k^{2}+m^{2}\right)^{-1} \widetilde{F}\right)^{-1}, \\
\delta H & =\delta \widetilde{H}\left(1-\left(k^{2}+m^{2}\right)^{-1} \widetilde{F}\right)^{-1} .
\end{aligned}
$$

Let us first proceed assuming this to be true. From eqs. (3.9) and (3.4) we see that the full propagator is given by

$$
\left(k^{2}+m^{2}\right)^{-1}+\left(k^{2}+m^{2}\right)^{-2} \widetilde{F}\left(1-\left(k^{2}+m^{2}\right)^{-1} \widetilde{F}\right)^{-1}=\left(k^{2}+m^{2}-\widetilde{F}(k)\right)^{-1} .
$$

If we choose a real basis of fields in position space then we have $\widetilde{F}(k)^{\dagger}=\widetilde{F}(k)$ and $\widetilde{F}(k)^{T}=\widetilde{F}(-k)$. In this case by choosing suitable unitary matrix $U(k)$ satisfying $U(-k)^{T}=$ $U(k)^{\dagger}$ we can express $\widetilde{F}(k)$ as $U(k) \widetilde{F}_{d}(k) U(k)^{\dagger}$ where $\widetilde{F}_{d}(k)$ is a diagonal matrix satisfying $\widetilde{F}_{d}(-k)=\widetilde{F}_{d}(k)$. Furthermore the genus expansion of $U(k)$ is free from poles at $k^{2}+m^{2}=0$ since $\widetilde{F}(k)$ has this property. We can now express (3.11) as

$$
U(k)\left(k^{2}+m^{2}-\widetilde{F}_{d}(k)\right)^{-1} U(k)^{\dagger}=U(k)\left(k^{2}+m^{2}-\widetilde{F}_{d}(k)\right)^{-1} U(-k)^{T} .
$$


Let $M_{p}^{2}$ denote the diagonal matrix that describes the locations of the zeroes of the eigenvalues of the diagonal matrix $k^{2}+m^{2}-\widetilde{F}_{d}(k)$ in the $-k^{2}$ plane. We can solve for this iteratively starting with the leading order solution $k^{2}=-m^{2}$. Then we can write

$$
\left(k^{2}+m^{2}-\widetilde{F}_{d}(k)\right)^{-1}=X_{d}(k)\left(k^{2}+M_{p}^{2}\right)^{-1},
$$

where $X_{d}(k)$ is a diagonal matrix whose genus expansion does not have any pole near $k^{2}=-m^{2}$ and satisfies $X_{d}(-k)=X_{d}(k)$. Defining

$$
Z^{1 / 2}(k)=U(k) \sqrt{X_{d}(k)},
$$

satisfying $Z^{1 / 2}(k)^{\dagger}=Z^{1 / 2}(-k)^{T}$ we can express the propagator (3.11) as

$$
Z^{1 / 2}(k)\left(k^{2}+M_{p}^{2}\right)^{-1} Z^{1 / 2}(-k)^{T} .
$$

The genus expansion of $Z^{1 / 2}(k)$ does not have any poles at $k^{2}+m^{2}=0$ since neither $U(k)$ nor $X_{d}^{1 / 2}(k)$ has such poles.

Now using eq. (3.9) we can express (3.10) as

$$
\delta H=\delta \widetilde{H}\left(1+\left(k^{2}+m^{2}\right)^{-1} F\right) .
$$

Comparing this with (3.8) we get

$$
\delta Y=\delta \widetilde{H} .
$$

Since $\delta \widetilde{H}$ does not have any pole near $k^{2}+m^{2}=0$ this establishes that $\delta Y$ also does not have any pole near $k^{2}+m^{2}=0$. This in turn establishes the desired result that the locations of the poles of (3.4) in the $k^{2}$ plane do not change under change in local coordinates.

\subsection{Explicit construction of $\widetilde{F}$ and $\delta \widetilde{H}$}

It now remains to prove the existence of pole free $\widetilde{F}$ and $\delta \widetilde{H}$ satisfying (3.9) and (3.10). We shall do this in steps.

1. First we extend the definitions of $\delta H$ and $F$ where we allow the external states inserted at the punctures (except at the special puncture) to be general string states of ghost number two ${ }^{7}$ inserted using the same local coordinate system as before. This makes $F$ into an infinite dimensional square matrix which we shall call $\mathcal{F}$ and $\delta H$ into an $n_{p} \times$ infinite dimensional matrix (since one of its two punctures is special) which we shall call $\delta \mathcal{H}$.

2. We now use another insight from string field theory [37]: it provides us with a triangulation of the moduli space in which the full moduli space of a genus $n$ Riemann surface with two punctures can be decomposed into a 'one particle irreducible' region $R_{n}$ and the rest. The region $R_{n}$ has the property that it does not contain any boundary of the moduli space in which a genus $n$ Riemann surface degenerates into a pair of two

\footnotetext{
${ }^{7}$ As will become clear later, we need to extend this definition only to those states which are annihilated by $L_{0}-\bar{L}_{0}, b_{0}$ and $\bar{b}_{0}$.
} 
punctured lower genus Riemann surfaces connected by a long handle, with each side containing one of the original punctures. The rest of the moduli space is obtained by gluing in all possible ways lower genus puctured Riemann surfaces corredponding to regions $R_{n^{\prime}}$ by the plumbing fixture procedure $[39,40]$. If we denote by $\widehat{\mathcal{F}}$ and $\delta \widehat{\mathcal{H}}$ the contributions to $\mathcal{F}$ and $\delta \mathcal{H}$ from integration over the one particle irreducible regions $R_{n}$ of the moduli spaces, then $\widehat{\mathcal{F}}$ and $\delta \widehat{\mathcal{H}}$ have no poles at $k^{2}+m^{2}=0$ since the region of integration does not include the degenerating Riemann surfaces. We shall shortly discuss how to define $\widehat{\mathcal{F}}$ and $\delta \widehat{\mathcal{H}}$ in the absence of a string field theory underlying the choice of local coordinates we have made. There is also an additional subtle point in the definition of $\delta \widehat{\mathcal{H}}$ which will be discussed in point 11 of this discussion.

3. We can regard $\widehat{\mathcal{F}}$ and $\mathcal{F}$ as maps from $\mathcal{H} \times \mathcal{H}$ to $\mathbb{C}$ where $\mathcal{H}$ denotes the space of string states of ghost number 2 . However since string states of ghost number 4 form the dual vector space of string states of ghost number 2 via the inner product in the CFT, we can also regard $\mathcal{F}$ and $\widehat{\mathcal{F}}$ as maps from states of ghost number 2 to string states of ghost number 4 . We shall in fact include left multiplication by the operator $\bar{b}_{0} b_{0}$ - the zero modes of the $b$ and $\bar{b}$ ghost fields — to regard $\mathcal{F}$ and $\widehat{\mathcal{F}}$ as maps from states of ghost number 2 to states of ghost number 2 . This is the way we shall interpret $\mathcal{F}$ and $\widehat{\mathcal{F}}$ from now on. By including similar factor in the definition of $\delta \mathcal{H}$ and $\delta \widehat{\mathcal{H}}$, they can be regarded as maps from string states of ghost number 2 to the space of special states.

4. With this convention the full contribution to $\mathcal{F}$ and $\delta \mathcal{H}$ is obtained by gluing $\widehat{\mathcal{F}}$ and $\delta \widehat{\mathcal{H}}$ using the string propagator

$$
\Delta=\frac{1}{4 \pi} \int_{0}^{2 \pi} d \theta \int_{0}^{\infty} d s e^{-s\left(L_{0}+\bar{L}_{0}\right)+i \theta\left(L_{0}-\bar{L}_{0}\right)}=\frac{1}{2} \delta_{L_{0}, \bar{L}_{0}} \int_{0}^{\infty} d s e^{-s\left(L_{0}+\bar{L}_{0}\right)} .
$$

The normalization of $\Delta$ has been chosen such that acting on special states at mass level $m$ it gives $\left(k^{2}+m^{2}\right)^{-1}$. We can now express $\mathcal{F}$ and $\delta \mathcal{H}$ as

$$
\begin{aligned}
\mathcal{F} & =\widehat{\mathcal{F}}+\widehat{\mathcal{F}} \Delta \widehat{\mathcal{F}}+\widehat{\mathcal{F}} \Delta \widehat{\mathcal{F}} \Delta \widehat{\mathcal{F}}+\cdots=\widehat{\mathcal{F}}(1-\Delta \widehat{\mathcal{F}})^{-1}=(1-\widehat{\mathcal{F}} \Delta)^{-1} \widehat{\mathcal{F}}, \\
\delta \mathcal{H} & =\delta \widehat{\mathcal{H}}+\delta \widehat{\mathcal{H}} \Delta \widehat{\mathcal{F}}+\delta \widehat{\mathcal{H}} \Delta \widehat{\mathcal{F}} \Delta \widehat{\mathcal{F}}+\cdots=\delta \widehat{\mathcal{H}}(1-\Delta \widehat{\mathcal{F}})^{-1} .
\end{aligned}
$$

Note that each factor of $\Delta$ is accompanied by a hidden factor of $\bar{b}_{0} b_{0}$ coming from $\widehat{\mathcal{F}}$; these are required to provide the correct integration measure on the moduli space. Eqs. (3.19) provide us with explicit implementation of plumbing fixture, building a higher genus Riemann surface from gluing of lower genus punctured Riemann surfaces.

In the world-sheet description, $\widehat{\mathcal{F}} \Delta \widehat{\mathcal{F}}$ contains integration over those Riemann surfaces, which can be obtained by gluing two Riemann surfaces corresponding to regions of the moduli space included in the definition of $\widehat{\mathcal{F}}$, at one each of their punctures by the relation

$$
w_{1} w_{2}=e^{-s+i \theta}, \quad 0 \leq s<\infty, \quad 0 \leq \theta<2 \pi,
$$

where $w_{1}$ and $w_{2}$ are the local coordinates at the punctures. Similar interpretation holds for terms like $\delta \widehat{\mathcal{H}} \Delta \widehat{\mathcal{F}}$. 
5. In the absence of an underlying string field theory we can use (3.19) to define $\widehat{\mathcal{F}}$ and $\delta \widehat{\mathcal{H}}$. Consider for example $\widehat{\mathcal{F}}$. Let $\mathcal{F}_{n}$ and $\widehat{\mathcal{F}}_{n}$ denote the genus $n$ contribution to $\mathcal{F}$ and $\widehat{\mathcal{F}}$ respectively. Since both $\mathcal{F}$ and $\widehat{\mathcal{F}}$ have genus expansion beginning at genus one, the genus expansion of (3.19) tells us that $\widehat{\mathcal{F}}_{1}$ is identical to $\mathcal{F}_{1}$. Now at genus two the right hand of the first equation in (3.19) gets a contribution from the $\widehat{\mathcal{F}}_{1} \Delta \widehat{\mathcal{F}}_{1}$ term. This represents integration over certain region of the genus two moduli space with the same integrand as that in the expression for $\mathcal{F}_{2}$. Then $\widehat{\mathcal{F}}_{2}$ is given by the integral of the same integrand over the complementary region of the genus two moduli space. The same process can now be repeated for higher genus, $\widehat{\mathcal{F}}_{n}$ being given by an integration over certain region of the genus $n$ moduli space with the same integrand as that of $\mathcal{F}_{n}$. The region of integration is the region that is not covered by gluing the lower genus $\widehat{\mathcal{F}}_{m}$ 's by $\Delta$. By construction $\widehat{\mathcal{F}}_{n}$ defined this way does not include integration over any region of the moduli space that corresponds to degeneration of the Riemann surface of the kind discussed before, since these regions are already included from the gluing of lower genus contributions. Since the structure of the second equation in (3.19) is similar to that of the first equation, the genus $n$ contribution to $\delta \widehat{\mathcal{H}}$ will be given by integration over the same region of the genus $n$ moduli space as that for $\widehat{\mathcal{F}}_{n}$, with the integrand being the same as that of $\delta \mathcal{H}$.

6. Note however that for this procedure to be consistent it is essential that for those Riemann surfaces which are built by gluing lower genus Riemann surfaces, represented in the genus expansion of the right hand side of (3.19) by product of lower genus contributions connected by $\Delta$, the choice of local coordinates at the punctures must coincide with those on the lower genus Riemann surfaces. We shall assume that the local coordinates have been chosen this way even if they are not inherited from an underlying string field theory. We also need to assume that the Riemann surfaces produced by the gluing procedure are all distinct, 1.e. the same Riemann surface should not be produced by two different gluing procedure. This can be achieved with an appropriate choice of local coordinates, e.g. by scaling the local coordinates by a sufficiently small number $\lambda$ we can ensure that the gluing produces only Riemann surfaces close to degeneration and hence different gluing produces different Riemann surfaces.

7. We define $P_{T}$ to be the projection operator into all states of momentum $k$ - physical and unphysical — with $L_{0}=\bar{L}_{0}=\left(k^{2}+m^{2}\right) / 4$, and define

$$
\begin{aligned}
\bar{\Delta} & \equiv \Delta-\frac{1}{k^{2}+m^{2}} P_{T} \\
\overline{\mathcal{F}} & \equiv \widehat{\mathcal{F}}+\widehat{\mathcal{F}} \overline{\mathcal{F}}+\widehat{\mathcal{F}} \bar{\Delta} \widehat{\mathcal{F}} \overline{\mathcal{F}}+\cdots=\widehat{\mathcal{F}}(1-\bar{\Delta} \widehat{\mathcal{F}})^{-1}=(1-\widehat{\mathcal{F}} \bar{\Delta})^{-1} \widehat{\mathcal{F}} \\
\delta \overline{\mathcal{H}} & \equiv \delta \widehat{\mathcal{H}}+\delta \widehat{\mathcal{H}} \bar{\Delta} \widehat{\mathcal{F}}+\delta \tilde{\mathcal{H}} \bar{\Delta} \widehat{\mathcal{F}} \bar{\Delta} \widehat{\mathcal{F}}+\cdots=\delta \widehat{\mathcal{H}}(1-\bar{\Delta} \widehat{\mathcal{F}})^{-1} .
\end{aligned}
$$

Physically $\overline{\mathcal{F}}$ and $\delta \overline{\mathcal{H}}$ denote 'one particle irreducible' contribution to appropriate two point functions of fields at mass level $m$ after integrating out the fields at other mass 
levels. Using (3.22) we can rewrite (3.19) as

$$
\begin{aligned}
\mathcal{F} & =\overline{\mathcal{F}}\left(1-\left(k^{2}+m^{2}\right)^{-1} P_{T} \overline{\mathcal{F}}\right)^{-1} \\
& =\overline{\mathcal{F}}+\overline{\mathcal{F}}\left(k^{2}+m^{2}\right)^{-1} P_{T} \overline{\mathcal{F}}+\overline{\mathcal{F}}\left(k^{2}+m^{2}\right)^{-1} P_{T} \overline{\mathcal{F}}\left(k^{2}+m^{2}\right)^{-1} P_{T} \overline{\mathcal{F}}+\cdots, \\
\delta \mathcal{H} & =\delta \overline{\mathcal{H}}\left(1-\left(k^{2}+m^{2}\right)^{-1} P_{T} \overline{\mathcal{F}}\right)^{-1} \\
& =\delta \overline{\mathcal{H}}+\delta \overline{\mathcal{H}}\left(k^{2}+m^{2}\right)^{-1} P_{T} \overline{\mathcal{F}}+\delta \overline{\mathcal{H}}\left(k^{2}+m^{2}\right)^{-1} P_{T} \overline{\mathcal{F}}\left(k^{2}+m^{2}\right)^{-1} P_{T} \overline{\mathcal{F}}+\cdots .
\end{aligned}
$$

8. We now define

$$
P=c_{1} \bar{c}_{1}\left|V_{i}\right\rangle\left\langle V_{i}\right| c_{-1} \bar{c}_{-1} c_{0} \bar{c}_{0} \otimes I_{z e r o},
$$

as the projection operator into the special states with tree level mass $m$. Here $I_{\text {zero }}$ corresponds to identity operator acting on the zero mode sector of non-compact bosons, labelled by $\left(k^{0}, \vec{k}\right)$. In the following we shall omit explicit mention of the operator $I_{z e r o}$ as the various operators we shall work with will always act as identity operator in this sector. Applying the projection operator $P$ on both sides of the first equation in (3.23) and from the right in the second equation in (3.23), and noting that

$$
P \mathcal{F} P=F, \quad \delta \mathcal{H} P=\delta H,
$$

we get

$$
\begin{aligned}
F=P \overline{\mathcal{F}} P & +P \overline{\mathcal{F}}\left(k^{2}+m^{2}\right)^{-1} P_{T} \overline{\mathcal{F}} P \\
& +P \overline{\mathcal{F}}\left(k^{2}+m^{2}\right)^{-1} P_{T} \overline{\mathcal{F}}\left(k^{2}+m^{2}\right)^{-1} P_{T} \overline{\mathcal{F}} P+\cdots, \\
\delta H=\delta \overline{\mathcal{H}} P & +\delta \overline{\mathcal{H}}\left(k^{2}+m^{2}\right)^{-1} P_{T} \overline{\mathcal{F}} P \\
& +\delta \overline{\mathcal{H}}\left(k^{2}+m^{2}\right)^{-1} P_{T} \overline{\mathcal{F}}\left(k^{2}+m^{2}\right)^{-1} P_{T} \overline{\mathcal{F}} P+\cdots .
\end{aligned}
$$

9. Now $P$ denotes projection operator into special states which transform in certain representations of the symmetry group $\mathrm{SO}(D) \times G . P_{T}-P$ denotes projection operator into states at the same mass level which are not special, and hence by definition transform in representations of $\mathrm{SO}(D) \times G$ other than those in which special states transform. Thus the two point function of special and non-special states on any Riemann surface vanishes, leading to $\left(P_{T}-P\right) \widehat{\mathcal{F}} P=0,\left(P_{T}-P\right) \Delta P=0$. This in turn gives

$$
\left(P_{T}-P\right) \overline{\mathcal{F}} P=0, \quad P \overline{\mathcal{F}}\left(P_{T}-P\right)=0 .
$$

Using this we can replace the $P_{T} \overline{\mathcal{F}} P$ factors in (3.26) by $P \overline{\mathcal{F}} P$. Defining

$$
\widetilde{F}=P \overline{\mathcal{F}} P, \quad \delta \widetilde{H}=\delta \overline{\mathcal{H}} P,
$$

we get

$$
\begin{aligned}
F & =\widetilde{F}+\widetilde{F}\left(k^{2}+m^{2}\right)^{-1} \widetilde{F}+\widetilde{F}\left(k^{2}+m^{2}\right)^{-1} \widetilde{F}\left(k^{2}+m^{2}\right)^{-1} \widetilde{F}+\cdots \\
\delta H & =\delta \widetilde{H}+\delta \widetilde{H}\left(k^{2}+m^{2}\right)^{-1} \widetilde{F}+\delta \widetilde{H}\left(k^{2}+m^{2}\right)^{-1} \widetilde{F}\left(k^{2}+m^{2}\right)^{-1} \widetilde{F}+\cdots
\end{aligned}
$$


10. This reproduces $(3.9),(3.10)$. Furthermore since $\overline{\mathcal{F}}$ and $\delta \overline{\mathcal{H}}$ have no poles at $k^{2}+m^{2}=$ 0 it follows that $\widetilde{F}$ and $\delta \widetilde{H}$ defined in (3.28) also have no poles at $k^{2}+m^{2}=0$. This is the desired result.

11. We now come to a subtle point in the definition of $\delta \widehat{\mathcal{H}}$ alluded to earlier. First consider the contributions $\delta \widehat{\mathcal{H}}$ to the right hand side of the second equation in (3.19). Naively, $\left(k^{2}+m^{2}\right) \delta \widehat{\mathcal{H}}$ represents the difference between two contributions, both given by integrating over the same subspace of the moduli space that is used to define $\widehat{\mathcal{F}}$. In one of them we use the original local coordinate encoded in the function $f$ at the puncture carrying momentum $k$, while in the other one we use the local coordinates encoded in the function $f+\delta f$ at the puncture carrying momentum $k$. This difference is clearly what we need to compute the contribution to $\left(k^{2}+m^{2}\right) \delta \mathcal{H}$ from these Riemann surfaces. For reasons that will become clear soon, let us denote this contribution to $\delta \widehat{\mathcal{H}}$ by $\delta_{0} \widehat{\mathcal{H}}$.

Now consider the contribution $\left(k^{2}+m^{2}\right) \delta_{0} \widehat{\mathcal{H}} \Delta \widehat{\mathcal{F}}$. Again this gives the difference between two contributions: $B-A$. The first contribution $A$ is obtained by gluing the Riemann surfaces corresponding to $\widehat{\mathcal{F}}$ to those corresponding to $\widehat{\mathcal{F}}$ at one each of their punctures using the original coordinate system $f$, with the coordinate at the external punctures also given by the original local coordinate system $f$. This induces a specific local coordinate system at the external punctures on the Riemann surfaces represented by $\widehat{\mathcal{F}} \Delta \widehat{\mathcal{F}}$ (see e.g. [42, 43]). By the compatibiity condition discussed in point 6 , this is the correct choice of coordinate system on the Riemann surfaces in the original system. The second contribution $B$ is obtained by gluing Riemann surfaces represented by $\widehat{\mathcal{F}}$ and $\widehat{\mathcal{F}}$ at one each of their punctures using the original coordinate system $f$, with the coordinate at the external puncture carrying momentum $k$ given by the deformed local coordinate system $f+\delta f$. This induces a specific local coordinate system at the external punctures carrying momentum $k$ on the Riemann surfaces represented by $\widehat{\mathcal{F}} \Delta \widehat{\mathcal{F}}$, but this is not the correct choice of coordinate system as prescribed in the deformed system since we are still using the original local coodinate system $f$ for the gluing. Let $f+\delta_{1} f$ denote the coordinate at the external puncture carrying momentum $k$ that we get using the gluing procedure described above and $f+\delta f$ be the local coordinate at the external puncture for the deformed system which we would get by using the coordinate system $f+\delta f$ both for external puncture and for the punctures we are using for gluing. ${ }^{8}$ Let us denote by $\left(k^{2}+m^{2}\right) \delta_{1} \widehat{\mathcal{H}}$ the difference between the two contributions, the $\left(k^{2}+m^{2}\right)$ factor being there due to the fact that the external vertex represents a dimension $(0,0)$ primary in the $k^{2}+m^{2} \rightarrow 0$ limit, and hence $f+\delta_{1} f$ and $f+\delta f$ acting on the external vertex gives the same result in the $k^{2}+m^{2} \rightarrow 0$ limit. Then $\left(k^{2}+m^{2}\right) \delta_{0} \widehat{\mathcal{H}} \Delta \widehat{\mathcal{F}}+\left(k^{2}+m^{2}\right) \delta_{1} \widehat{\mathcal{H}}$ gives

\footnotetext{
${ }^{8}$ Two points may need clarification here. First we are using the same symbol $f$ for the coordinates on the component Riemann surface and the Riemann surface we get by gluing these components since $f$ stands for the original choice of local coordinates on all Riemann surfaces. Similar remark applies to $f+\delta f$. The second point is that while comparing the coordinate systems $f+\delta f$ and $f+\delta_{1} f$ we work at the same point in the moduli space of the glued Riemann surface.
} 
the desired difference between the off-shell amplitudes computed using the deformed system and the original system. We can then add the error term $\delta_{1} \widehat{\mathcal{H}}$ to $\delta_{0} \widehat{\mathcal{H}}$ to define a corrected $\delta \widehat{\mathcal{H}}$ so that the net contribution to $\delta \mathcal{H}$ can still be written as the right hand side of the second equation in (3.19). The only possible caveat with this is that since the definition of $\delta_{1} \widehat{\mathcal{H}}$ involves integration over moduli spaces of Riemann surfaces corresponding to $\widehat{\mathcal{F}} \Delta \widehat{\mathcal{F}}$, this involve a degeneration limit where the parameter $s$ appearing in the definition of $\Delta$ in (3.18) goes to $\infty$. Integration over $s$ from this region could produce a pole at $k^{2}=-m^{2}$. We shall now argue that this does not happen. For this note that in the $s \rightarrow \infty$ limit the Riemann surface degenerates into two Riemann surfaces, and the local coordinates induced at external punctures are inherited from the local coordinates at the external punctures of the Riemann surfaces which are being glued, and independent of the local coordinates at the punctures which we use to glue the two Riemann surfaces. Thus the functions $f+\delta f$ and $f+\delta_{1} f$ should become identical as $s \rightarrow \infty$. From this we conclude that for large $s$ they should differ by a term proportional to $q=e^{-s+i \theta}$. As a result the expression for $\delta_{1} \widehat{\mathcal{H}}$, which involves difference in the contributions with local coordinates $f+\delta_{1} f$ and $f+\delta f$ at the external puncture carrying momentum $k$, will have an extra factor of $q$ and/or $\bar{q}$ in the integrand. Since the leading contribution to the integrand in (3.18) in the $s \rightarrow \infty$ limit comes from states of mass level $m$ and is proportional to $e^{-\left(k^{2}+m^{2}\right) s / 2} \sim|q|^{\left(k^{2}+m^{2}\right) / 2}$, we see that an extra factor of $q$ and/or $\bar{q}$ in the integrand will kill the pole at $k^{2}=-m^{2}$. Thus $\delta_{1} \widehat{\mathcal{H}}$ is free from poles at $k^{2}+m^{2}=0$.

To summarize, $\left(k^{2}+m^{2}\right)\left(\delta_{1} \widehat{\mathcal{H}}+\delta_{0} \widehat{\mathcal{H}} \Delta \widehat{\mathcal{F}}\right)$, added to $P \widehat{\mathcal{F}} \Delta \widehat{\mathcal{F}}$, produces correctly the contribution to off-shell Green's function with the deformed coordinate system from those Riemann surfaces which correspond to $\widehat{\mathcal{F}} \Delta \widehat{\mathcal{F}}$. Furthermore $\delta_{1} \widehat{\mathcal{H}}$ does not contain any pole at $k^{2}=-m^{2}$. Defining $\delta \widehat{\mathcal{H}}=\delta_{0} \widehat{\mathcal{H}}+\delta_{1} \widehat{\mathcal{H}}$, we ensure the equality of two sides of the second equation of (3.19) to this order. We can then move on to the term $\left(\delta_{1} \widehat{\mathcal{H}}+\delta_{0} \widehat{\mathcal{H}} \Delta \widehat{\mathcal{F}}\right) \Delta \widehat{\mathcal{F}}$ and carry out similar analysis, generating further correction $\delta_{2} \widehat{\mathcal{H}}$ to $\delta \widehat{\mathcal{H}}$. After carrying out this procedure to the desired order in perturbation theory we can ensure that (3.19) and hence all subsequent equations still hold with this new definition of $\delta \widehat{\mathcal{H}}$.

\section{S-matrix elements}

The on-shell S-matrix element for massive external string states can be analyzed by following a procedure similar to the one used for mass renormalization. Again we shall restrict to S-matrix elements of special states (and possibly massless states for which there is no mass renormalization); the S-matrix elements of other states can be found in principle from the above by computing its residues at appropriate poles. Using the given local coordinate system for $n$-punctured Riemann surfaces we compute the off-shell $n$-point function $F_{a_{1} \cdots a_{n}}^{(n)}\left(k_{1}, \cdots k_{n}\right)$ of $n$ external legs. With the help of $(2.4),(2.1)$ we can then define the 
on-shell S-mtatrix elements via

$$
\begin{aligned}
S_{a_{1} \cdots a_{n}}^{(n)}\left(k_{1}, \cdots k_{n}\right)= & \lim _{k_{i}^{2} \rightarrow-m_{a_{i}, p}^{2}} F_{b_{1} \cdots b_{n}}^{(n)}\left(k_{1}, \cdots k_{n}\right) \times \\
& \quad \times \prod_{i=1}^{n}\left\{Z_{i}^{-1 / 2}\left(k_{i}\right)_{a_{i} b_{i}}\left(k_{i}^{2}+m_{a_{i}, p}^{2}\right)\left(k_{i}^{2}+m_{a_{i}}^{2}\right)^{-1}\right\} .
\end{aligned}
$$

We shall now prove that $S^{(n)}$ defined this way is invariant under a change of local coordinates even though $F^{(n)}$ 's themselves transform under such changes. The change in $S^{(n)}$ comes from two sources: the change in $F^{(n)}$ and the change in $Z_{i}^{-1 / 2}\left(k_{i}\right)$. We begin by computing the change in $Z_{i}^{-1 / 2}\left(k_{i}\right)$. First of all comparing (2.3) with the transformation law (3.5) of the propagator under a change of local coordinates, we get

$$
\delta Z_{i}^{1 / 2}\left(k_{i}\right)=\delta Y_{i}\left(k_{i}\right) Z_{i}^{1 / 2}\left(k_{i}\right)
$$

where $\delta Y_{i}$ is the same as $\delta Y$ introduced in (3.5) and computed in (3.17) for the $i$-th external state. The multiplication on the right hand side of (4.2) should be regarded as a matrix multiplication. This gives

$$
\delta Z_{i}^{-1 / 2}\left(k_{i}\right)=-Z_{i}^{-1 / 2}\left(k_{i}\right) \delta Y_{i}\left(k_{i}\right)=-Z_{i}^{-1 / 2}\left(k_{i}\right) \delta \widetilde{H}_{i}\left(k_{i}\right),
$$

where in the last step we have used the equality of $\delta Y$ and $\delta \widetilde{H}(k)$ given in (3.17).

Next we shall analyze the contribution to $\delta F_{b_{1} \cdots b_{n}}^{(n)}$. This can be expressed as

$$
\delta F_{b_{1} \cdots b_{n}}^{(n)}=\sum_{j} \delta_{j} F_{b_{1} \cdots b_{n}}^{(n)},
$$

where $\delta_{j}$ denotes the effect of the change of local coordinates at the $j$-th puncture. We shall later show that there exist quantities $\widetilde{F}_{j ; b_{1} \cdots b_{n}}^{(n)}$ and $\delta_{j} \widetilde{H}_{b_{1} \cdots b_{n}}^{(n)}$ whose perturbation expansions have no poles at $k_{j}^{2}+m_{a_{j}}^{2}=0$ and in terms of which we have the relations

$$
F_{b_{1} \cdots b_{n}}^{(n)}=\left(1-\left(k_{j}^{2}+m_{a_{j}}^{2}\right)^{-1} \widetilde{F}_{j}\left(k_{j}\right)\right)_{b_{j} c_{j}}^{-1} \widetilde{F}_{j, b_{1} \cdots b_{j-1} c_{j} b_{j+1} \cdots b_{n}}^{(n)},
$$

and

$$
\begin{aligned}
\delta_{j} F_{b_{1} \cdots b_{j}}^{(n)}=\left(k_{j}^{2}+m_{a_{j}}^{2}\right)\left[\delta_{j} \widetilde{H}_{b_{1} \cdots b_{n}}^{(n)}+\delta \widetilde{H}_{j}\left(k_{j}\right)_{b_{j} c_{j}}\right. & \left(1-\left(k_{j}^{2}+m_{a_{j}}^{2}\right)^{-1} \widetilde{F}_{j}\left(k_{j}\right)\right)_{c_{j} d_{j}}^{-1} \\
& \left.\left(k_{j}^{2}+m_{a_{j}}^{2}\right)^{-1} \widetilde{F}_{j, b_{1} \cdots b_{j-1} d_{j} b_{j+1} \cdots b_{n}}^{(n)}\right],
\end{aligned}
$$

where the quantities $\widetilde{F}_{j}\left(k_{j}\right)$ and $\delta \widetilde{H}_{j}\left(k_{j}\right)$ are the same matrices which were called $\widetilde{F}\left(k_{j}\right)$ and $\delta \widetilde{H}\left(k_{j}\right)$ in eqs. $(3.9),(3.10)$, with the subscript $j$ indicating that we have to use appropriate matrices $\left(\widetilde{F}_{j}\left(k_{j}\right)\right)_{b_{j} c_{j}}$ and $\left(\delta \widetilde{H}_{j}\left(k_{j}\right)\right)_{b_{j} c_{j}}$ relevant for the $j$-th external leg. The various products and inverses appearing in (4.5), (4.6) are then interpreted as matrix products and matrix inverses acting on the $j$-th leg.

We shall prove the existence of $\widetilde{F}_{j ; b_{1} \cdots b_{n}}^{(n)}$ and $\delta_{j} \widetilde{H}_{b_{1} \cdots b_{n}}^{(n)}$ with the desired properties later; for now we shall proceed assuming this to be true. Using (4.5) we can express (4.6) as

$$
\delta_{j} F_{b_{1} \cdots b_{j}}^{(n)}=\left(k_{j}^{2}+m_{a_{j}}^{2}\right) \delta_{j} \widetilde{H}_{b_{1} \cdots b_{n}}^{(n)}+\delta \widetilde{H}_{j}\left(k_{j}\right)_{b_{j} c_{j}} F_{b_{1} \cdots b_{j-1} c_{j} b_{j+1} \cdots b_{n}}^{(n)} .
$$


We are now in a position to calculate $\delta S^{(n)}$. Using eqs. (4.1), (4.3) and (4.7) we get

$$
\begin{aligned}
& \delta S_{a_{1} \cdots a_{n}}^{(n)}=\lim _{k_{i}^{2} \rightarrow-m_{a_{i}, p}^{2} \forall i} \sum_{j=1}^{n} \prod_{\substack{\ell=1 \\
\ell \neq j}}^{n}\left\{Z_{\ell}^{-1 / 2}\left(k_{\ell}\right)_{a_{\ell} b_{\ell}}\left(k_{\ell}^{2}+m_{a_{\ell}, p}^{2}\right)\left(k_{\ell}^{2}+m_{a_{\ell}}^{2}\right)^{-1}\right\} \\
& \times\left(k_{j}^{2}+m_{a_{j}, p}^{2}\right)\left(k_{j}^{2}+m_{a_{j}}^{2}\right)^{-1} \\
& \times\left[\delta Z_{j}^{-1 / 2}\left(k_{j}\right)_{a_{j} b_{j}} F_{b_{1} \cdots b_{j}}^{(n)}+Z_{j}^{-1 / 2}\left(k_{j}\right)_{a_{j} b_{j}} \delta_{j} F_{b_{1} \cdots b_{n}}^{(n)}\right] \\
& =\lim _{k_{i}^{2} \rightarrow-m_{a_{i}, p}^{2} \forall i} \sum_{j=1}^{n} \prod_{\ell=1}^{n}\left\{Z_{\ell}^{-1 / 2}\left(k_{\ell}\right)_{a_{\ell} b_{\ell}}\left(k_{\ell}^{2}+m_{a_{\ell}, p}^{2}\right)\left(k_{\ell}^{2}+m_{a_{\ell}}^{2}\right)^{-1}\right\} \\
& \times \sum_{j=1}^{n}\left[-\delta \widetilde{H}_{j}\left(k_{j}\right)_{b_{j} c_{j}} F_{b_{1} \cdots b_{j-1} c_{j} b_{j+1} \cdots b_{n}}^{(n)}+\left(k_{j}^{2}+m_{a_{j}}^{2}\right) \delta_{j} \widetilde{H}_{b_{1} \cdots b_{n}}^{(n)}\right. \\
& \left.+\delta \widetilde{H}_{j}\left(k_{j}\right)_{b_{j} c_{j}} F_{b_{1} \cdots b_{j-1} c_{j} b_{j+1} \cdots b_{n}}^{(n)}\right] \\
& =\lim _{k_{i}^{2} \rightarrow-m_{a_{i}, p}^{2} \forall i} \prod_{\ell=1}^{n}\left\{Z_{\ell}^{-1 / 2}\left(k_{\ell}\right)_{a_{\ell} b_{\ell}}\left(k_{\ell}^{2}+m_{a_{\ell}, p}^{2}\right)\right\} \sum_{j=1}^{n} \prod_{\substack{\ell=1 \\
\ell \neq j}}^{n}\left(k_{\ell}^{2}+m_{a_{\ell}}^{2}\right)^{-1} \delta_{j} \widetilde{H}_{b_{1} \cdots b_{\ell}}^{(n)} .
\end{aligned}
$$

Now note that the genus expansion of the $j$-th term in the sum has no poles at $k_{j}^{2}+m_{a_{j}}^{2}=0$ since there is no explicit factor of $\left(k_{j}^{2}+m_{a_{j}}^{2}\right)^{-1}$ and the genus expansion of $\delta_{j} \widetilde{H}^{(n)}$ does not contain any poles at $\left(k_{j}^{2}+m_{a_{j}}^{2}\right)=0$. As a result after resummation this term will have no pole at $k_{j}^{2}+m_{a_{j}, p}^{2}=0$, and after being multiplied by the $\left(k_{j}^{2}+m_{a_{j}, p}^{2}\right)$ term, will give vanishing contribution in the $k_{j}^{2} \rightarrow-m_{a_{j}, p}^{2}$ limit. Since this analysis can be repeated for every $j$, we see that $\delta S^{(n)}$ vanishes. Thus the S-matrix is invariant under a change in the local coordinates.

It remains to prove the existence of $\widetilde{F}_{j ; b_{1} \cdots b_{n}}^{(n)}$ and $\delta_{j} \widetilde{H}_{b_{1} \cdots b_{n}}^{(n)}$ satisfying (4.5), (4.6) and having no poles at $k_{j}^{2}=-m_{a_{j}}^{2}$ in their genus expansion. For this we first define $\mathcal{F}_{j}^{(n)}$ by allowing the $j$-th external state of $F^{(n)}$ to be an arbitrary string state. We also use the fact that the change in local coordinates generates a vertex proportional to $\left(k^{2}+m^{2}\right)$ to introduce the quantity $\delta_{j} H^{(n)}$ via

$$
\delta_{j} H_{b_{1} \cdots b_{n}}^{(n)}=\left(k_{j}^{2}+m_{a_{j}}^{2}\right)^{-1} \delta_{j} F_{b_{1} \cdots b_{n}}^{(n)} .
$$

Then in the same spirit as the $\widehat{\mathcal{F}}$ and $\delta \widehat{\mathcal{H}}$ defined in (3.19) we introduce $\widehat{\mathcal{F}}_{j}^{(n)}$ and $\delta_{j} \widehat{H}^{(n)}$ via the expansion:

$$
\begin{aligned}
\mathcal{F}_{j}^{(n)} & =\widehat{\mathcal{F}}_{j}^{(n)}+\widehat{\mathcal{F}} \Delta \widehat{\mathcal{F}}_{j}^{(n)}+\widehat{\mathcal{F}} \Delta \widehat{\mathcal{F}} \Delta \widehat{\mathcal{F}}_{j}^{(n)}+\cdots=(1-\widehat{\mathcal{F}} \Delta)^{-1} \widehat{\mathcal{F}}_{j}^{(n)}, \\
\delta_{j} H^{(n)} & =\delta_{j} \widehat{H}^{(n)}+\delta \widehat{\mathcal{H}}_{j} \Delta \widehat{\mathcal{F}}_{j}^{(n)}+\delta \widehat{\mathcal{H}}_{j} \Delta \widehat{\mathcal{F}} \Delta \widehat{\mathcal{F}}_{j}^{(n)}+\delta \widehat{\mathcal{H}}_{j} \Delta \widehat{\mathcal{F}} \Delta \widehat{\mathcal{F}} \Delta \widehat{\mathcal{F}}_{j}^{(n)}+\cdots \\
& =\delta_{j} \widehat{H}^{(n)}+\delta \widehat{\mathcal{H}}_{j} \Delta(1-\widehat{\mathcal{F}} \Delta)^{-1} \widehat{\mathcal{F}}_{j}^{(n)}
\end{aligned}
$$

where $\widehat{\mathcal{F}}$ has been defined via (3.19) and $\delta \widehat{\mathcal{H}}_{j}$ is the same as $\delta \widehat{\mathcal{H}}$ defined in (3.19), but for the $j$-th external state. All multiplications in (4.10) are matrix multiplications on the $j$-th 
external leg with fixed indices $b_{i}$ for $i \neq j$ on all other legs. $\widehat{\mathcal{F}}_{j}^{(n)}$ and $\delta_{j} \widehat{H}^{(n)}$ represent contributions to $\mathcal{F}_{j}^{(n)}$ and $\delta_{j} H^{(n)}$ which are one particle irreducible on the $j$-th external leg. Thus they are given by integration over subregions of the moduli space of Riemann surface with the same integrands as $\mathcal{F}^{(n)}$ and $\delta_{j} H^{(n)}$, and these subregions have the property that they do not include any degeneration of the $j$-th external leg. ${ }^{9}$ Thus the genus expansions of $\widehat{\mathcal{F}}_{j}^{(n)}$ and $\delta_{j} \widehat{H}^{(n)}$ do not have any pole at $k_{j}^{2}+m_{a_{j}}^{2}=0$. Now we define

$$
\begin{aligned}
\overline{\mathcal{F}}_{j}^{(n)} & =\widehat{\mathcal{F}}_{j}^{(n)}+\widehat{\mathcal{F}} \bar{\Delta} \widehat{\mathcal{F}}_{j}^{(n)}+\widehat{\mathcal{F}} \bar{\Delta} \widehat{\mathcal{F}} \bar{\Delta} \widehat{\mathcal{F}}_{j}^{(n)}+\cdots=(1-\widehat{\mathcal{F}} \bar{\Delta})^{-1} \widehat{\mathcal{F}}_{j}^{(n)} \\
\delta_{j} \widetilde{H}^{(n)} & =\delta_{j} \widehat{H}^{(n)}+\delta \widehat{\mathcal{H}}_{j} \bar{\Delta} \widehat{\mathcal{F}}_{j}^{(n)}+\delta \widehat{\mathcal{H}}_{j} \bar{\Delta} \widehat{\mathcal{F}} \bar{\Delta} \widehat{\mathcal{F}}_{j}^{(n)}+\delta \widehat{\mathcal{H}}_{j} \bar{\Delta} \widehat{\mathcal{F}} \bar{\Delta} \widehat{\mathcal{F}} \bar{\Delta} \widehat{\mathcal{F}}_{j}^{(n)}+\cdots \\
& =\delta_{j} \widehat{H}^{(n)}+\delta \widehat{\mathcal{H}}_{j} \bar{\Delta}(1-\widehat{\mathcal{F}} \bar{\Delta})^{-1} \widehat{\mathcal{F}}_{j}^{(n)}=\delta_{j} \widehat{H}^{(n)}+\delta \widehat{\mathcal{H}}_{j}(1-\bar{\Delta} \widehat{\mathcal{F}})^{-1} \bar{\Delta} \widehat{\mathcal{F}}_{j}^{(n)},
\end{aligned}
$$

where $\bar{\Delta}$ has been defined in (3.21). Since $\bar{\Delta}$ has no poles at $k_{j}^{2}+m_{a_{j}}^{2}=0$, the genus expansions of $\overline{\mathcal{F}}_{j}^{(n)}$ and $\delta_{j} \widetilde{H}^{(n)}$ also do not have any poles at $k_{j}^{2}+m_{a_{j}}^{2}=0$. Using (3.22), (4.10) and (4.11) we get

$$
\begin{aligned}
\mathcal{F}_{j}^{(n)} & =\left(1-\overline{\mathcal{F}}\left(k_{j}^{2}+m_{a_{j}}^{2}\right)^{-1} P_{T}\right)^{-1} \overline{\mathcal{F}}_{j}^{(n)} \\
\delta_{j} H^{(n)} & =\delta_{j} \widetilde{H}^{(n)}+\delta \overline{\mathcal{H}}_{j}\left(k_{j}^{2}+m_{a_{j}}^{2}\right)^{-1} P_{T}\left(1-\overline{\mathcal{F}}\left(k_{j}^{2}+m_{a_{j}}^{2}\right)^{-1} P_{T}\right)^{-1} \overline{\mathcal{F}}_{j}^{(n)} .
\end{aligned}
$$

We now define

$$
\widetilde{F}_{j}^{(n)}=P \overline{\mathcal{F}}_{j}^{(n)} .
$$

Since the genus expansion of $\overline{\mathcal{F}}_{j}^{(n)}$ has no poles at $k_{j}^{2}=-m_{a_{j}}^{2}$, the genus expansion of $\widetilde{F}_{j}^{(n)}$ also has no poles at $k_{j}^{2}=-m_{a_{j}}^{2}$. It follows from the definition of special states that $\delta \overline{\mathcal{H}}_{j} P_{T}=\delta \overline{\mathcal{H}}_{j} P$. Using this and (4.13), multiplying the first equation of (4.12) by $P$ from the left, using $P \mathcal{F}_{j}^{(n)}=F^{(n)}$ and eqs. (3.27), (3.28) we can write the two equations in $(4.12)$ as

$$
\begin{aligned}
F^{(n)} & =\left(1-\left(k_{j}^{2}+m_{a_{j}}^{2}\right)^{-1} \widetilde{F}_{j}\right)^{-1} \widetilde{F}_{j}^{(n)}, \\
\delta_{j} H^{(n)} & =\delta_{j} \widetilde{H}^{(n)}+\delta \widetilde{H}_{j}\left(k_{j}^{2}+m_{a_{j}}^{2}\right)^{-1}\left(1-\left(k_{j}^{2}+m_{a_{j}}^{2}\right)^{-1} \widetilde{F}_{j}\right)^{-1} \widetilde{F}_{j}^{(n)} .
\end{aligned}
$$

This reproduces (4.5) and (4.6) after using (4.9).

\section{Discussion and generalizations}

In this paper we have given an algorithm for computing renormalized mass and S-matrix elements for a special class of massive states in bosonic string theory, and have shown that these are independent of the specific off-shell continuation that we use for computing them. While the results are in the same spirit as the proof of gauge invariance of physical mass and S-matrix elements in a gauge theory, in many sense the analysis here is simpler than in gauge theories. In the latter the gauge invariance results from cancellation between the

\footnotetext{
${ }^{9}$ The definition of $\delta_{j} \widehat{H}^{(n)}$ suffers from subtleties of the same kind that affects the definition of $\delta \widehat{\mathcal{H}}$, and these are dealt with in the same way as in the case of $\delta \widehat{\mathcal{H}}$, following the procedure discussed in point 11 at the end of section 3 .
} 
contributions from different Feynman diagrams, while here we do not require any such cancellations. In fact if we had been trying to prove gauge invariance of renormalized mass and S-matrix elements in string field theory, we would still need cancellation between different Feynman diagrams. ${ }^{10}$

The simplicity in string theory of course is a consequence of the fact that in string theory there is only one contribution from every genus. Technically the difference between our analysis and the corresponding analysis in string field theory can be traced to the fact that in string field theory a change in local coordinates will change the local coordinates not only at the external punctures, but also at the internal punctures that we use to glue two Riemann surfaces using the plumbing fixture procedure. As a result each Feynman diagram gets additional contribution from the change in local coordinates at the internal punctures which cancel between different Feynman diagrams.

Clearly there are many generalizations of our analysis that are needed. We expect that for special vertex operators our analysis can be generalized in a straightforward manner to heterotic and superstring theories. Consider bosonic states coming from the NS sector (in heterotic string theory) or NS-NS sector (in type II string theories) — for Ramond sector we expect similar analysis to go through with the propagator (3.18) acquiring extra numerator factors [1]. In this case the choice of local coordinates at the punctures will have to be replaced by a choice of local superconformal coordinates. On-shell vertex operators are independent of local superconformal coordinates, and hence under a change of local coordinates off-shell vertex operators change by a term proportional to $\left(k^{2}+m^{2}\right)$ as in the case of bosonic string theory. We also have the analog of the gluing relations (3.20) (see e.g. [1]) and hence the relations (3.19) with the bosonic propagator $\Delta$ given by the same expression as (3.18). ${ }^{11}$ Thus we expect that extending our analysis to superstring and heterotic string theories is straightforward.

For general external states we expect new complications even in the bosonic string theory. This is due to the fact that under quantum correction the physical states would begin mixing with the unphysical states and we need to take into account this mixing for defining an appropriate off-shell continuation. For example from genus two onwards $\widehat{\mathcal{F}}$ will have non-zero matrix element between a physical state and a BRST trivial state from the boundary of the region of integration of the moduli space that defines $\widehat{\mathcal{F}}$, forcing us to change the definition of the physical state. Furthermore the required mixing will depend on the particular off-shell continuation we choose 1.e. on the choice of local coordinates at the punctures. We expect that once these effects are taken into account, we shall be able to directly prove that the renormalized mass and S-matrix elements are independent of the off-shell continuation for all physical states, suitably defined.

\footnotetext{
${ }^{10}$ A change of local coordinates correspond to a field redefinition of the string field [41] followed by a gauge transformation that is needed to bring the transformed fields to the Siegel gauge.

${ }^{11}$ By choosing the local coordinate system appropriately we can ensure that the splitting of the moduli space we have used, e.g. in defining $\widehat{\mathcal{F}}$ etc, requires only information about the region of the moduli space near the boundary where complications arising out of non-splitness of the supermoduli space are not present [17]. For example by scaling the function $f\left(w ; z_{0}\right)$ introduced at the beginning of section 3 to $f\left(\lambda w ; z_{0}\right)$ for sufficiently small $\lambda$, we can ensure that $\widehat{\mathcal{F}}$ includes the contribution from most of the region of the moduli space except those close to the boundaries where the Riemann surface splits apart into two lower genus surfaces.
} 
In fact it seems to us that the off-shell formalism could be a useful way of studying string perturbation theory both for massive and massless external states, and can be used to give alternate proofs of well known results in string theory. For example in the standard on-shell approach the proof of decoupling of pure gauge states, corresponding to trivial elements of the BRST cohomology, involves first showing that the result is given by a total derivative in the moduli space and then showing that the boundary terms arising from the integration of the total derivative terms vanish. In the off-shell formalism the boundary terms can be ignored altogether since they can be made to vanish by appropriate off-shell continuation of the external momenta. The price we pay is that due to BRST noninvariance of the external off-shell states there will be additional terms proportional to one or more powers of $\left(k_{i}^{2}+m_{a_{i}}^{2}\right)$ associated with the external states. In individual terms these may be cancelled by inverse powers of $\left(k_{i}^{2}+m_{a_{i}}^{2}\right)$ coming from integration over moduli near the boundaries. Thus the task will be to show that the final result vanishes nevertheless in the on-shell limit.

\section{Acknowledgments}

We thank Rajesh Gopakumar, Michael Green and Barton Zwiebach for useful discussions. The work of R.P. and A.S. was supported in part by the DAE project 12-R\&D-HRI-5.020303. A.R. was supported by the Ramanujan studentship of Trinity College, Cambridge and would like to thank HRI, Allahabad for hospitality during the initial stages of this work. The work of A.S. was also supported in part by the J. C. Bose fellowship of the Department of Science and Technology, India.

Open Access. This article is distributed under the terms of the Creative Commons Attribution License (CC-BY 4.0), which permits any use, distribution and reproduction in any medium, provided the original author(s) and source are credited.

\section{References}

[1] E. Witten, Superstring perturbation theory revisited, arXiv:1209.5461 [INSPIRE].

[2] A. Belopolsky, De Rham cohomology of the supermanifolds and superstring BRST cohomology, Phys. Lett. B 403 (1997) 47 [hep-th/9609220] [INSPIRE].

[3] A. Belopolsky, New geometrical approach to superstrings, hep-th/9703183 [INSPIRE].

[4] A. Belopolsky, Picture changing operators in supergeometry and superstring theory, hep-th/9706033 [INSPIRE].

[5] E. D'Hoker and D.H. Phong, Two loop superstrings. 1. Main formulas, Phys. Lett. B 529 (2002) 241 [hep-th/0110247] [INSPIRE].

[6] E. D'Hoker and D.H. Phong, Two loop superstrings. 2. The chiral measure on moduli space, Nucl. Phys. B 636 (2002) 3 [hep-th/0110283] [INSPIRE].

[7] E. D'Hoker and D.H. Phong, Two loop superstrings. 3. Slice independence and absence of ambiguities, Nucl. Phys. B 636 (2002) 61 [hep-th/0111016] [INSPIRE]. 
[8] E. D'Hoker and D.H. Phong, Two loop superstrings 4: The cosmological constant and modular forms, Nucl. Phys. B 639 (2002) 129 [hep-th/0111040] [INSPIRE].

[9] E. D'Hoker and D.H. Phong, Two-loop superstrings. V. Gauge slice independence of the N-point function, Nucl. Phys. B 715 (2005) 91 [hep-th/0501196] [inSPIRE].

[10] E. D'Hoker and D.H. Phong, Two-loop superstrings VI: Non-renormalization theorems and the 4-point function, Nucl. Phys. B 715 (2005) 3 [hep-th/0501197] [INSPIRE].

[11] E. D'Hoker and D.H. Phong, Two-loop superstrings. VII. Cohomology of chiral amplitudes, Nucl. Phys. B 804 (2008) 421 [arXiv:0711.4314] [inSPIRE].

[12] E. Witten, Notes on supermanifolds and integration, arXiv:1209.2199 [INSPIRE].

[13] E. Witten, Notes on super riemann surfaces and their moduli, arXiv:1209.2459 [INSPIRE].

[14] E. Witten, More on superstring perturbation theory, arXiv:1304.2832 [INSPIRE].

[15] E. Witten, Notes on holomorphic string and superstring theory measures of low genus, arXiv:1306.3621 [INSPIRE].

[16] E. Witten, The feynman it in string theory, arXiv:1307.5124 [INSPIRE].

[17] R. Donagi and E. Witten, Supermoduli space is not projected, arXiv:1304.7798 [INSPIRE].

[18] S. Weinberg, Radiative corrections in string theory, in The Oregon Meeting, Proceedings of the Annual Meeting of the Division of Particles and Fields of the APS, Eugene, Oregon, 1985, R.C. Hwa ed., World Scientific, Singapore (1986).

[19] N. Seiberg, Anomalous dimensions and mass renormalization in string theory, Phys. Lett. B 187 (1987) 56 [InSPIRE].

[20] H. Ooguri and N. Sakai, String loop corrections from fusion of handles and vertex operators, Phys. Lett. B 197 (1987) 109 [inSPIRE].

[21] H. Yamamoto, One loop mass shifts in $O(32)$ open superstring theory, Prog. Theor. Phys. 79 (1988) 189 [INSPIRE].

[22] A. Sen, Mass renormalization and BRST anomaly in string theories, Nucl. Phys. B 304 (1988) 403 [inSPIRE].

[23] S.R. Das, Renormalizing handles and holes in string theory, Phys. Rev. D 38 (1988) 3105 [INSPIRE].

[24] S.-J. Rey, Unified view of BRST anomaly and its cancellation in string amplitudes, Nucl. Phys. B 316 (1989) 197 [inSPIRE].

[25] B. Sundborg, Selfenergies of massive strings, Nucl. Phys. B 319 (1989) 415 [InSPIRE].

[26] N. Marcus, Unitarity and regularized divergences in string amplitudes, Phys. Lett. B 219 (1989) 265 [inSPIRE].

[27] K. Amano and A. Tsuchiya, Mass splittings and the finiteness problem of mass shifts in the type II superstring at one loop, Phys. Rev. D 39 (1989) 565 [INSPIRE].

[28] C.J.H. Lee, BRST anomalies and mass renormalization with anomalous $\mathrm{U}(1)$ gauge symmetries in string theory, UMI-92-24830 (1992) [INSPIRE].

[29] A. Berera, The mass renormalization of string theory, Phys. Rev. D 49 (1994) 6674 [INSPIRE]. 
[30] L. Del Debbio, E. Kerrane and R. Russo, Mass corrections in string theory and lattice field theory, Phys. Rev. D 80 (2009) 025003 [arXiv:0812.3129] [INSPIRE].

[31] D. Chialva, String mass shifts, Nucl. Phys. B 819 (2009) 225 [arXiv:0903.3979] [INSPIRE].

[32] P.C. Nelson, Covariant insertion of general vertex operators, Phys. Rev. Lett. 62 (1989) 993 [INSPIRE].

[33] A.G. Cohen, G.W. Moore, P.C. Nelson and J. Polchinski, An off-shell propagator for string theory, Nucl. Phys. B 267 (1986) 143 [InSPIRE].

[34] A.G. Cohen, G.W. Moore, P.C. Nelson and J. Polchinski, Semi off-shell string amplitudes, Nucl. Phys. B 281 (1987) 127 [inSPIRE].

[35] L. Álvarez-Gaumé, C. Gomez, G.W. Moore and C. Vafa, Strings in the operator formalism, Nucl. Phys. B 303 (1988) 455 [inSPIRE].

[36] L. Álvarez-Gaumé, C. Gomez, P.C. Nelson, G. Sierra and C. Vafa, Fermionic strings in the operator formalism, Nucl. Phys. B 311 (1988) 333 [INSPIRE].

[37] B. Zwiebach, Closed string field theory: quantum action and the B-V master equation, Nucl. Phys. B 390 (1993) 33 [hep-th/9206084] [inSPIRE].

[38] N. Berkovits, Y. Okawa and B. Zwiebach, WZW-like action for heterotic string field theory, JHEP 11 (2004) 038 [hep-th/0409018] [INSPIRE].

[39] P. Di Vecchia et al., $N$ point $g$ loop vertex for a free bosonic theory with vacuum charge $Q$, Nucl. Phys. B 322 (1989) 317 [inSPIRE].

[40] J. Polchinski, Factorization of bosonic string amplitudes, Nucl. Phys. B 307 (1988) 61 [INSPIRE].

[41] H. Hata and B. Zwiebach, Developing the covariant Batalin-Vilkovisky approach to string theory, Annals Phys. 229 (1994) 177 [hep-th/9301097] [INSPIRE].

[42] A. LeClair, M.E. Peskin and C.R. Preitschopf, String field theory on the conformal plane. 1. Kinematical principles, Nucl. Phys. B 317 (1989) 411 [INSPIRE].

[43] A. LeClair, M.E. Peskin and C.R. Preitschopf, String field theory on the conformal plane. 2. Generalized gluing, Nucl. Phys. B 317 (1989) 464 [InSPIRE]. 